survival between cTnI $>0.06$ and $<0.5 \mu \mathrm{g} / 1$ and $c T n I \geq 0.5 \mu \mathrm{g} / 1$ subgroups. Increased mortality (HR, 95\% CI) was associated with ages 70 to 80 years $(2.58,1.17$ to 3.91$)$ and $>80$ years $(3.30,3.50$ to $5.06)$, peripheral vascular disease $(1.50,1.16$ to 1.94$)$, heart failure (1.36, 1.05 to 1.83$)$, diabetes $(1.68,1.36$ to 2.07$)$, severe LV dysfunction $(1.50,1.00$ to 2.21$)$ and creatinine per $10 \mu \mathrm{mol} / 1$ (1.65, 1.02 to 1.08 ), whereas ages 50 to 60 years $(0.55,0.32$ to 0.96$), \beta$ blockers $(0.53,0.44$ to 0.64$)$, aspirin (0.80 0.65 to 0.99), ACE inhibitors $(0.67,0.56$ to 0.80$)$, statins $(0.73,0.59$ to 0.90$)$ and revascularisation $(0.33,0.12$ to 0.92$)$ lowered the risk of death.

Conclusion Quantitative evaluation of cTnI concentration in ACS patients with a cTnI $>0.06 \mu \mathrm{g} / \mathrm{l}$ is associated with no added prognostic information. However, the dichotomisation of patients by cTnI status ("positive" and "negative") continues to facilitate ACS risk stratification.

\section{P1-477 RELATIONSHIP BETWEEN PREGNANCY OR CHILDBIRTH SATISFACTION AND WILLINGNESS FOR SUBSEQUENT PREGNANCY IN JAPAN}

doi:10.1136/jech.2011.142976g.66

\begin{abstract}
${ }^{1} \mathrm{~A}$ Nagai, ${ }^{1} \mathrm{~T}$ Tanaka, ${ }^{2} \mathrm{~K}$ Matsuura, ${ }^{3} \mathrm{Y}$ Yamazaki, ${ }^{4} \mathrm{~T}$ Ojima, ${ }^{5} \mathrm{~K}$ Tamakoshi, ${ }^{6} \mathrm{M}$ Arakida, ${ }^{7} \mathrm{~K}$ Ichikawa. ${ }^{1}$ University of Yamanashi, Chuo, Yamanashi, Japan; ${ }^{2}$ Fukuoka Prefectural University, Tagawa, Fukuoka, Japan; ${ }^{3}$ Aichi children's Health and Medical center, Obu, Aichi, Japan; ${ }^{4}$ Hamamatsu University School of Medicine, Hamamatsu, Sizuoka, Japan; ${ }^{5}$ Nagoya University, Nagoya, Aichi, Japan; ${ }^{6}$ International University of Health and Welfare, Odawara, Kanagawa, Japan; ${ }^{7} J a p a n e s e ~ M i d w i v e s '$ Association, Taito-ku, Tokyo, Japan
\end{abstract}

Introduction In Japan, a survey of children and their parents was conducted to assess national campaign "Sukoyaka Family 21" in 2009. Our aim was to clarify the relationship between pregnancy or childbirth satisfaction and willingness for subsequent pregnancy.

Methods Children aged 3-4 months, 18 months, or 3 years who underwent medical check-up in 138 cities and their mothers were surveyed. Data from 5500 children aged 3-4 months and their mothers was analysed; this data included responses regarding willingness for subsequent pregnancy and pregnancy or childbirth satisfaction. Factor analysis was performed to verify existing categories of 15 items associated with pregnancy or childbirth satisfaction. Multivariate logistic regression analyses were performed to determine factors associated with the willingness for subsequent pregnancy, stratified by mothers' age and parity.

Results Factor analysis indicated that 4 factors influence pregnancy or childbirth satisfaction: medical care, family support, birthing facility, and support in the workplace and society. Multivariate logistic regression analyses in mothers having one child revealed that those aged 25-29 years and satisfied with the birthing facility [OR: 2.26, 95\% CI: 1.06 to 4.85 ] and those aged 30-34 [OR: 2.21, 95\% CI 1.12 to 4.35 ] or $>35$ years [OR: $2.94,95 \%$ CI 1.15 to 7.51 ] and satisfied with family support were significantly more willing for subsequent pregnancy. Among mothers having $>2$ children, no significant association was found.

Conclusion Factors that influence willingness for subsequent pregnancy vary with mother's age. Therefore, age should be considered when planning services and support for parturient women.

\section{P1-478 PRE-HOSPITAL DELAY AND PREDICTORS OF MORTALITY IN PATIENTS PRESENTING TO EMERGENCY DEPARTMENT WITH STROKE: A DEVELOPING NATION SCENARIO}

doi:10.1136/jech.2011.142976g.67

V Nagaraja, ${ }^{*}$ G Bhat, M A Khan. MMC \& Rl, Karnataka, India

Background Stroke is one of the leading causes of death and disability in developed nations.
Materials and Methods A prospective study was conducted to assess the factors influencing this delay in admission of acute stroke cases, in-hospital mortality was calculated and factors associated with mortality were looked for. Survival was calculated using KaplanMeier curve and Cox-proportional hazards model was constructed to identify independent predictors of inpatient mortality.

Results Of 134 subjects studied, 73.4\% were male with mean (SD) age of 53.8(18.0) years. Median delay in reaching hospital was $9 \mathrm{~h}$ and only 18 (13.4\%) reached hospital within $3 \mathrm{~h}$ and $31(20.3 \%)$ reached within $6 \mathrm{~h}$. Distances from hospital, referral, belief in myths and alternate medicine and low threat perception of symptoms of stroke were independent factors associated with delay in arrival. Living in city, day time onset, urgency shown by attendant, availability of transport and presence of family history were associated with early arrival. ICF rate has no relation with age $(p=0.516)$, sex $(p=0.460)$, number of episodes (0.795), underlying hypertension $(p=0.905)$. Odds of diabetics dying were 12 times higher than nondiabetics. Inpatient mortality was also significantly higher in smokers compared with non-smokers $(p=0.004)$, in patients with right-sided compared with left-sided hemiplegic $(p=0.029)$ and who couldn't afford computed tomography (CT) scan ( $p=0.007)$.

Conclusion Majority of patients did not reach hospital early enough to receive thrombolytics and had high in-hospital mortality. Diabetes and smoking were independent predictors of in-hospital mortality.

\section{P1-479 IODINE DEFICIENCY IN CHILDREN: A COMPARATIVE STUDY IN THE TWO DISTRICTS OF SOUTH-INTERIOR KARNATAKA}

doi:10.1136/jech.2011.142976g.68

V Nagaraja, * M A Khan, G Bhat. MMC \& RI, Mysore, Karnataka, India

Introduction and Objective Iodine is an essential micronutrient. A daily consumption of $100-150 \mu \mathrm{g}$ of Iodine is recommended for normal human growth and development. Iodine Deficiency Disorders (IDD) can be easily prevented. Simplest, most effective and most inexpensive preventive method is consumption of iodated salt. In this study, we estimated the prevalence of IDD among children in the rural areas of Mysore and Coorg districts, and the results were compared.

Materials and Methods A cross sectional study in the age group of 6-12 years using PPS systematic sampling method. The total sample size being 10082 out of which 5337 is from Mysore and the rest from Coorg district. Clinical examination of the thyroid was done and urine samples were collected for Iodine estimation. Data were analysed using SAS software.

Results and Conclusion Prevalence of Goitre was more in females compared to males with a ratio of 2:1 in Mysore districts and 4:1 in Coorg district. It was observed that IDD is Endemic in both districts and the presence of goitre is directly proportional to the age of children. The significant difference in the level of Goitre in children across districts and within districts is being studied.

\section{P1-480 VALIDATION OF A FOOD FREOUENCY QUESTIONNAIRE TO ASSESS DIET IN CHILDREN AGED 4-5 YEARS}

doi:10.1136/jech.2011.142976g.69

${ }^{1,2} \mathrm{~J}$ Vioque, ${ }^{1,2} \mathrm{E}$ M Navarrete-Muñoz, ${ }^{* 1,2} \mathrm{D}$ Gimenez, ${ }^{2,3} \mathrm{M}$ Rebagliato, ${ }^{2,3} \mathrm{~F}$ Ballester, ${ }^{2,3} \mathrm{C}$ Iñiguez, ${ }^{4} \mathrm{~F}$ Granado, ${ }^{1,2} \mathrm{M} \mathrm{G}$ de la Hera, ${ }^{1,2} \mathrm{M}$ Pastor-Valero. ${ }^{1}$ Miguel Hernández University, Elche-Alicante, Spain; ${ }^{2}$ CIBER Epidemiología y Salud Pública (CIBERESP), Madrid, Spain; ${ }^{3}$ Centro Superior de Investigación en Salud Pública, Valencia, Spain; ${ }^{4}$ Hospital Universitario Puerta de Hierro, Madrid, Spain

Introduction We need valid food frequency questionnaires (FFQ) to assess diet in preschool children using parental report. 